\title{
Entrevista a R. M. Hare: a propósito de Ordenando la ética
}

\author{
JOSEP-MARIA TERRICABRAS Y JOAN VERGÉS GIFRA \\ Cátedra Ferrater Mora de Pensamiento Contemporáneo \\ Universidad de Girona
}

Indudablemente R. M. Hare es uno de los filósofos morales más importantes e influyentes del panorama filosófico de los últimos tiempos. A él debemos la formulación más poderosa de la teoría ética del prescriptivismo, una tradición de pensamiento sobre el lenguaje moral que, según Hare, puede remontarse a Aristóteles, Kant y Mill. Pero no tan sólo eso. Su teoria ética está estrechamente vinculada a una concepción moral normativa de carácter utilitarista. Acerca de ella, James Griffin, uno de los más destacados estudiosos del utilitarismo, escribió una vez: «[la teoría de Harc] constituye la explicación del utilitarismo más rigurosa y lúcida de nuestro siglo" (J. Griffin, «Modern Utilitarianism», Revue Internationale de Philosophie, núm. 141, 1982, p. 353).

Eso no impide, sin embargo, que Hare sea también uno de los filósofos morales más controvertidos. La entrevista que reproducimos a continuación pretende contribuir tanto a destacar la importancia de su obra como a subrayar algunos de los aspectos polémicos de sus propuestas teóricas. Creemos, en particular, que esta entrevista puede servir como un buen complemento al libro Ordenando la ética (Ariel, 1999), que ha aparecido recientemente en nuestras librerías *.

Pregunta.-Su incursión en teoría ética en los años cincuenta fue motivada por el deseo de contrarrestar las implicaciones irracionalistas del emotivismo - la concepción predominante de aquel entonces-. ¿Piensa usted que el irracionalismo sigue siendo el problema más importante de la filosofia de hoy en día?

Respuesta.- $-S t$, en la medida en que hay filosofos irracionalistas.

P.-Tendrá que admitir, sin embargo, que el racionalismo no parece estar de moda actualmente. En realidad, uno podría pensar que empieza a haber un cierto consenso a la hora de destacar los «limites" de la razón en ética ( $B$. Williams, I. Berlin). Algunos filósofos sostienen que la filosofía moral debería ser «menos ambiciosa» de lo que ha venido siendo hasta ahora (J. Griffin). Otros recusan los inten-

* Dos aclaraciones y un agradecimiento: en primer lugar, cabe decir que la publicaciōn de Ordenando la ética ha sido posible gracias al amable ofrecimiento para su traducción que el propio Hare hizo recientemente a la Cátedra Ferrater Mora -institución en la cual estaba previsto que diera unas lecciones a principios de los noventa que al final, desgraciadamente, tuvieron que suspenderse por problemas de salud-. En segundo lugar, debemos precisar que la presente entrevista se ha llevado a cabo, primero, a través de un intercambio epistolar, y posteriormente, especialmente los detalles, a través de un contacto personal. El hecho de que muchas respuestas se hayan fraguado en un intercambio escrito explica que en la entrevista haya un número notable de referencias bibliográficas - que no hemos eliminado por el interés que podrán tencr para aquellos interesados en la obra de Hare-. Aprovechamos la ocasión, finalmente, para agradecer muy sinceramente el esfuerzo, la constancia y el interés que el prof. Hare ha demostrado tener en ambas colaboraciones, sin olvidar la inestimable ayuda de su mujer, Catherine. 
tos de querer "racionalizar la razón" y al mismo tiempo defienden abiertamente la necesidad de abandonar completamente el enfoque racionalista en filosofía (R. Rorty). ¿Qué opina usted al respecto?

$R$ - L L a verdad es que nunca he entendido qué concepciones defiende en realidad Williams, aunque reconozco que sus críticas a otros filósofos son poderosas. Berlin fue mejor historiador de las ideas que filósofo; en concreto, nunca comprendió qué sucede en los conflictos morales, por eso creyó que era imposible resolverlos mediante la razón. Respecto a Rorty, no estoy al tanto de su obra más reciente (no se puede leer todo y, por tanto, uno debe limitarse a la lectura de aquellos autores que prometen ser iluminadores).

P.-Usted afirma que la discusión pacífica entre las culturas es posible gracias a la existencia común de palabras valorativas tales como «debería $(O E, 1.7)$ * Ahora bien, usted saca esta conclusión a partir de un análisis lingüístico de una lengua en concreto, el inglés. ¿No sería posible, pues, objetarle que la validez de sus conclusiones se limita tan sôlo a una lengua o, como mucho, a una cultura en particular?

R.-Respondo a esta cuestión al principio del libro, en el primer capitulo, a lo cual tengo poco que añadir. Mi teoría se basa en mi comprensión de las palabras morales del inglés, en tanto que hablante nativo de esta lengua, una comprensión que creo que otros hablantes del inglés comparten conmigo. Pero si estuviera equivocado, aun asi habría proporcionado una explicación correcta de las cuestiones que planteo. En este punto me remito a $G$. E. Moore, cuando al comienzo de Principia Ethica dice: «Por consiguiente, usaré la palabra l"bueno" o "bien"] en el sentido en que creo se usa esta palabra nomalmente; pero al mismo tiempo no estoy ansioso por discutir si tengo razón o no al pensar que se usa así. Yo me ocupo tan sólo de aquel objeto o idea al cual, en mi opinión, suele referirse esa palabra, tenga o no tenga razón para creerlo asís (\$6). Así pues, otros filósofos tal vez planteen preguntas distintas a las mías, pero no lo creo. En cualquier caso, a menudo es dificil saber qué cuestiones creen estar planteando. Por otro lado, es verdad que existen palabras distintas en lenguas distintas y que se puede emprender una investigación empínica para estudiarlas. Pero ése no es trabajo de filósofos. Además, uno debe tener clara la distinción que existe entre opiniones morales sustanciales distintas y usos de las palabras morales distintos; un mismo uso puede ser empleado para defender opiniones distintas, para elogiar formas de vida distintas, por ejemplo. Yo no acepto lo que parece decir MacIntyre, que los cambios en el uso de las palabras morales y los cambios en las opiniones morales tienen que ir juntos, uno al lado del otro.

P.-De acuerdo. Déjenos intentar, de todos modos, plantear el mismo problema de antes desde otra perspectiva. El uso que hacemos del lenguaje moral es un hecho contingente, parece. Las conclusiones que obtengamos de su análisis serán, por tanto, indicativas de cómo usamos el lenguaje moral nosotros actualmente. Tal vez haya, pues, otros usos posibles de las palabras morales. ¿Cómo podríamos determinar, pues, cuál de ellos es preferible?

R.-iClaro que puede haber otros usos de las palabras morales, pero entonces ya no expresarín los juicios morales en el mismo sentido! Humpty Dumpty -el personaje de Alicia en el pais de las maravillas-tiene razón y «todos», por ejemplo, podría significar to mismo que ahora significa kalgunos», $y$ edeberias podría significar también otra cosa. Podemos usar las palabras a nuestro

* Abreviamos así el libro Ordenando la ética. La numeración que sigue a $O E$ corresponde al número de una sección del libro. 
antojo. Ahora bien, si usamos las palabras de un modo distinto a como las usamos al plantearnos una cuestión en particular, entonces ya no estaremos planteando la misma cuestion. Y lo que a mi me interesa son los problemas morales relacionadus con el uso actual -y no otro- de la palabra «deberia».

P.-En este punto quizá ayudaría a aclarar un poco el asunto que nos fijáramos en su método. Aparentemente su método es deductivo en el siguiente sentida: primero tenemos que conocer el significado general de las palabras morales y luego sabremos qué podemos decir en los casos particulares en que su uso parece necesario; primero debemos saber en qué casos es incorrecto matar a alguien en general, por ejemplo, y luego podremos decidir en qué otros casos particulares es incorrecto terminar con la vida de una persona ( $O E$, 2.2). En este sentido, parece desmarcarse de otras propuestas metodológicas de carácter más bien holístico que no requieren este tipo de claridad inicial en la investigación (Rawls, por ejemplo, en Una teoría de la justicia). ¿Definiria su método como fundacionalista?

R.-En mi áltimo libro, que ha salido hace poco, Objective Prescriptions (Oxford UP, 1999), trato esta cuestión. En un capitulo llamado *Fundacionalismo y coherentismo en éticas, distingo entre el fundacionalismo cartesiano, que yo rechazo, y el fundacionalismo kantiano al que se refiere el tífulo mismo de la Fundamentación de la metafísica de las costumbres, y con el cual estoy de acuerdo. Obviamente podemos llegar a una comprension de los significados y la lógica de las palabras morales ambulando, al usarlas. Pero hasta que no tengamos claro su significado no seremos capaces de responder las cuestiones morales racionalmente. Rawls, en mi opinión, consigue esta comprensión tan sólo parcialmente y de una forma poco clara.
P.-Pasemos ahora, si le parece, a cuestiones relacionadas con su análisis del lenguaje moral. Según usted, los enunciados morales tienen dos características: la prescriptividad y la universalizabilidad. Ésa es la principal conclusión de su análisis del lenguaje moral y, probablemente, una de las contribuciones más importantes de su filosofía moral a la historia de la filosofía contemporánea. Los enunciados morales son prescripciones universales. Cuando alguien dice que se debería hacer $x$ en $y$ se compromete a actuar en consecuencia (si es que habla sinceramente), y a defender este compromiso en todas las circunstancias similares a $y$ en sus propiedades universales, y ello independientemente de quién se halle en la situación y. La conclusión de su análisis, sin embargo, ha recibido muchas críticas. En particular, se ha criticado con especial vehemencia la tesis de que es un hecho del lenguaje moral el que los enunciados morales deban ser uniwersalizables. La universalizabilidad de los enunciados morales parece ser una característica sustantiva, no formal, del lenguaje moral. Existe la sospecha, como usted mismo reconoce ( $O E, 1.8$ ), de que su trabajo sea un ejercicio de ilusionismo $y$ de que nos esté intentando hacer creer que pueden salir conejos de la chistera. Por eso, también existe la sospecha de que su teoría ética no satisface el requisito de neutralidad que usted mismo exige para cualquier teoria ética $(O E, 6.8)$.

R.-Esta crítica es importante. Quienes me critican con más acierto en esta cuestión. son Simon Blackburn (Spreading the Word, Oxford UP, 1984) y mi hijo, John Hare (The Moral Gap, Oxford UP, 1996), que están libres de las confusiones que sufren muchos otros filósofos. Blackbum discute lo que el llama «deberes esmorales» (shmoral) y mi hijo hace una distinción entre diferentes tipos de universalizabilidad (un juicio de prudencia, por ejemplo, es universalizable con respecto a los agentes pero no con res- 
pecto a los destinatarios). Pero como en el libro trato ya este problema, quizá no sea necesario repetir el argumento (OE, 5.8).

$P$.-He aqui una pregunta que no parece resuelta en el libro y que, de algún modo, está relacionada con la cuestión anterior. Concedamos que el uso actual del lenguaje moral exige por parte del hablante un cierto compromiso con respecto a la posibilidad de universalizar los enunciados morales. Aun así, una cosa es decir que el lenguaje moral se utiliza de un modo determinado y otra cosa es explicar por qué se utiliza de ese modo, o qué razones hay para utilizarlo de ese modo. Con esto abordamos el tema de cómo motivar a la gente para que utilice el lenguaje moral. En su libro, justamente ahí donde ahora nos remitia, usted mismo parece reconocer que aún no ha resuelto este problema $(O E, 5.8)$. Pero éste es un problema crucial para dar credibilidad a su intento. ¿No?

R.- Cómo motivan. Esta cuestión nos conduce al problema que a menudo se plantea confusamente con la pregunta «¿́Por qué deberia ser moral?». En realidad, aquí existen como mínimo dos cuestiones: 1) iPor qué deberia usar el lenguaje moral?, y 2) ¿Por qué debería actuar de acuerdo con las prescripciones a las que me veo llevado si razono de acuerdo con su lógica - la lógica del lenguaje moral-? Ustedes me formulan la primera pregunia. La respuesta a esta pregunta es que tal lenguaje consiste en un modo indispensable de plantear y responder cuestiones que la mayoría de nosotros queremos plantear. La respuesta a 2) es que está en nuestro interés, en general, hacerlo así. En otro libro (Moral Thinking, $11.3,12.6)$ he argumentado que si fuéramos a educar a un niño y consideráramos tan sólo su interés, deberiamos educarlo de tal modo que fuera a utilizar el lenguaje moral tal como yo lo analizo, con sus dos niveles.

P.-Otra cuestión. Usted distingue claramente en los enunciados morales dos tipos distintos de significado: el significado evaluativo o prescriptivo (susceptible de constituir una lógica del lenguaje moral) y el significado descriptivo (consistente en las condiciones de aplicación de las palabras morales). Ello le permite explicar tanto el hecho del desacuerdo moral como la posibilidad del acuerdo moral, puesto que mientras que el significado descriptivo varía en función de las particularidades de cada cultura, el significado prescriptivo permanece invariable. En este punto, sin embargo, surge la sospecha de si' tal distinción no será demasiado tajante. A veces parecería que las opiniones morales, o el significado descriptivo, puede incidir en el uso que se hace de las palabras morales y modificar su significado evaluativo. Tal vez si una persona religiosa que ha basado todo su comportamiento en los dogmas «descubre» de repente que Dios no existe termine creyendo que «debería» no significa nada más que «el capellán quiere que yo haga...».

R.-Es cierto que un cambio en las opiniones morales puede terminar en un cambio en los significados descriptivos de las palabras morales; pero no es menos cierto que el significado evaluativo puede seguir siendo el mismo. Está claro que "deberia podría terminar significando wel capellain quiere que yo haga..."-como sucede en realidad en boca de algunas personas que utilizan el lenguaje moral en su uso no comendatorio (non-commendatory use) - Lvéase al respecto $m i$ The Language of Morals, 7.5, 11.1 ss.]. Pero tal uso no es central, puesto que no podria existir sin el uso prescriptivo, que si es crucial para el lenguaje moral.

P.-Su teoría ética está compuesta de dos elementos: uno formal y el otro sustancial $(O E, 4.7)$. El elemento formal consiste en el análisis lógico de las palabras morales, en especial de la palabra *deberíam. El elemento sustancial consiste en la aplicación de la lógica del lenguaje moral al mundo, $y$ en especial a los hechos que 
afectan a las preferencias de la gente que lo puebla. En su opinión, tan sólo una pequeña parte de las dificultades que se presentan para resolver problemas morales prácticos tienen que ver con aspectos formales o lógicos; la gran mayoría (el 99 por 100 apunta en $O E, 2.5$ ) de las dificultades tienen que ver con los hechos: el problema mayor, en la práctica, es que la gente se ponga de acuerdo con respecto a los hechos del mundo. Pero alguien podría dudar de que ello sea así y de que podamos dejar de lado tan fácilmente las dificultades sustanciales. ¿No forma parte de la deliberación moral misma el proceso de discriminación de los hechos relevantes?

R.-Claro que si. Pero resolver las dificultades factuales no es una tarea filosófica. El mejor modo de abordar estas dificultades es asociándose con aquellos que han estudiado los hechos - los médicos en el caso de la ética médica, por ejemplo-. El filosofo tiene que hacer su propia contribución, consistente en clarificar los problemas. Esta tarea del filósofo tiene tanta importancia como la otra, tal como yo mismo he podido comprobar participando en muchos grupos de trabajo relacionados con la ética médica. Casi la mitad de mis trabajos recientes están dedicados a cuestiones morales prácticas, y siempre he introducido los estudiantes a la filosofia mediante el planteamiento de cues. tiones de este tipo. En cualquier caso, sin embargo, Ordenando la ética es un libro sobre ética teórica. Quien estuviera interesado en un tratamiento más detallado de mi respuesta podría leer el capitulo 5, «Filosofia y conflicton, de Objective Prescriptions.

P.-De todos modos, ahora nos gustaría seguir el hilo de la antcrior pregunta. Puesto que, según su teoría, los hechos más relevantes para la moral son los hechos que afectan las preferencias de la gente, limitémonos, en principio, a los problemas que existen para identificar claramente esas preferencias. Según su teoría, cuando un agente delibera adecuadamente acerca de la corrección de una acción, tiene que considerar las consecuencias que su acción tendrá o podría tener sobre las otras personas. Eso significa que tiene que ponerse en la piel de cada una de las personas afectadas y determinar si podría querer, con sus preferencias en mente, que esa acción se llevara a cabo. El problema es muy parecido al problema que tiene un solo individuo a la hora de decidir qué preferencias personales quiere satisfacer. Por ese camino, su teoría se convierte en una versión peculiar de utilitarismo. Ahora bien, ies siempre posible identificarse con las otras personas de este modo, es decir, sentir sus preferencias como si fueran propias?

$R$ - Es obvio que el pensamiento moral es dificil, $y$ en algunos casos extremos imposible. Cualquier teoria que lo convierta en algo fácil debe estar equivocada. Y está claro que una de las cosas más dificiles acerca del pensamiento moral es ponerse en la situación de las otras personas. 2 Pero he sugerido jamás otra cosa? Tenemos que admitir que no somos arcángeles - o sea, seres con poderes superhumanos, con un conocimiento superhumano-, pero debemos intentar comportamos lo mejor que podamos utilizando el nivel intuitivo. Si el problema al que ustedes apuntan es el del escepticismo sobre las mentes de las otras personas, o la cuestión sobre la posibilidad de realizar comparaciones interpersonales de preferencias, entonces debo responder lo siguiente. Con respecto a lo primero, no creo que tal escepticismo sea plausible. Además, el hecho de que los têrminos uyos y utus no tengan un contenido descriptivo, en sentido estricto, penmite resolver el problema sobre el sentido de hacer enunciados sobre los estados mentales de las demás personas. Con respecto a lo segundo, creo que mi teoría permite realizar comparaciones interpersonales de preferencias. Pero trato estas cuestiones con mucho más detalle en mi libro Moral Thinking, 7.3 yss. 
P.-Desearíamos concretar mejor nuestras dudas mediante un ejemplo. Supongamos que una persona está considerando si debería castigar o no a alguien por el mal que éste le ha hecho. Supongamos que tales consideraciones tienen sentido independientemente de la conveniencia de que existan sanciones sociales para que se respeten las reglas de convivencia - como si los dos individuos se encontraran en un estado de naturaleza lockeano, o como si estas dos personas estuvieran solas en el mundo-. En esta situación no parece que el juicio determinante fuera precisamente acerca de las preferencias del destinatario de la acción. Porque lo importante de un castigo es que a nadie le gusta ser castigado. ¿No serían más importantes aquí consideraciones de mérito, consideraciones de derecho, etc.? El mismo problema surge con respecto al problema de si una persona debería estar a favor o en contra de la pena de muerte. Aquí tampoco parece jugar ningún papel la idea de ponerse en la piel del otro.

R.-Si pensamos en los intereses de la sociedad en general, el criminal sale ganando al ser castigado de acuerdo con un sistema legal justo. En relación con esta cuestión la solución de los «dos niveles» es útil. Esta solución nos permite resolver la antinomia representada en las dos citas de Kant con las que empieza el capítulo 8 de Ordenando la ética. Aun cuando el fin supremo sea wla felicidad de toda la humanidad, en la práctica el mejor modo de realizar tal fin consiste en tener reglas de justicia -reglas de justicia penal, en particular-y atenerse a ellas (aunque no necesariamente el tipo de reglas draconianas que Kant justificó). Con respecto a la pena de muerte, yo estoy en contra de tal medida en los países civilizados, porque no existen pruebas suficientes de que sea un buen recurso disuasivo y además es irreversible: en demasiados casos se ha ejecutado a gente inocente. Ahora bien, en segun qué paises tal medida quizá sea la línica pena posible para determinados crimenes (por ejemplo, donde no haya prisiones seguras, o donde la situación política sea inestable). Tal vez Milosevic debería ser ejecutado por sus crimenes contra la humanidad, al igual que los lideres nazis. Todo esio puede apoyarse en razones de tipo utilitarista.

P.-Veamos otro ejemplo, ahora relacionado con unos supuestos efectos perjudiciales de su recurso de identificación con las preferencias de las otras personas. Supongamos, como no parece improbable que sea, que haya casos de free riders (o aprovechados) no perjudiciales: porque el acto es insignificante para el funcionamiento normal de las instituciones sociales, porque se trata de un caso completamente aislado, etc. ¿No podría alguien querer universalmente ser un free rider mientras sus actos de free riding no fueran perjudiciales para el funcionamiento normal de la sociedad, aceptando que cuando los bienes públicos estuvieran en peligro él y todo el mundo estaría obligado a participar en su mantenimiento? Se trạtaría de una sociedad de pequeños tramposos, por decirlo así, $y$ no parece haber ninguna razón para pensar que no podría funcionar bien en conjunto.

$R$-Buena pregunta. Mi respuesta al problema del free riding - basada en una importante tesis doctoral de mi alumna de Florida, Rory Weiner-es que necesitamos principios de justicia para determinar la medida de nuestra contribución al bien común, incluyendo en él el bien de los miembros más débiles de la sociedad mundial. $Y$ qué principios de justicia deberiamos adoptar tal vez cambie de una sociedad a otra en función de las circunstancias de tales sociedades. Los principios de justicia, pues, son relativos a las circunstancias de las sociedades. Tienen que seleccionarse al nivel intuitivo del pensamiento moral. Ahora bien, principios de justicia diferentes, adecuados para condiciones diferentes en sociedades diferentes, todos ellos pteden ser justificados 
en el nivel critico en base a razones utilitaristas mediante la pregunta: Lqué principios deberia adoptar tal sociedad a fin de maximizar la satisfacción de preferencias de la sociedad mundial en general, contando con que cada individuo vale uno? Es improbable que una sociedad de pequeños tramposos llegue a ser una sociedad feliz.

P.-De acuerdo con su respuesta parecc que la idea de derechos humanos debe justificarse en términos de satisfacción de preferencias.

R.-El modo de determinar qué derechos tienen las personas es mediante la pregunta: ¿qué principios morales sobre derechos son tales que su observación y respeto conduciria a la máxima satisfacción de preferencias de todas ellas, consideradas imparcialmente? Todos los juicios sobre derechos, como todos los juicios sobre justicia, tienen lugar en el nivel intuativo, a excepción del derecho a una igual consideracion y respeto, que puede ser establecido en el nivel critico. Todo esto es consistente con mi teoria utilitarista. Estoy bastante de acuerdo con el libro de J. Wayne Summer sobre la fundamentación moral de los derechos (The Moral Foundation of Rights, Oxford UP, 1987).

P.-En sus respuestas a las ültimas preguntas usted se refiere más de una vez a la distinción entre niveles del pensamiento moral y al carácter utilitarista de su teoría. A continuación, si le parece bien, nos gustaría concentrarnos en estas dos cuestiones. Veamos primero la distinción entre niveles del pensamiento moral. En su opinión, el pensamiento moral tiene lugar a dos niveles: a nivel intuitivo y a nivel crítico. La mayoria de las veces, en nuestra cotidianeidad, nos movemos en el nivel intuitivo. Otras veces, sin embargo, tenemos que pensar a nivel crítico. Pero, icómo pasamos de un nivel a otro? ¿Dónde termina un nivel y empieza el otro?

R-Los limites entre los dos niveles quedarán claros si nos fijamos en las transi- ciones del uno al otro. La foma mâs común , por la cual nos vemos forzados a ascender al nivel crítico es cuando nuestras intuiciones sobre un caso particular entran en conflicto y cuando, por consiguiente, si permaneciésemos en el nivel intuitivo, nos veriamos obligados a decir que deberiamos, ast como que no deberiamos, actuar de un modo determinado. Sin embargo, no hay ningún mal en criticar nuestras intuiciones, siempre $y$ cuando tal cosa no nos conduzca a la tentación de cuestionarlo todo. Si a partir de una intuición apelamos a otras, icómo sabremos que estas segundas son mejores que la primera?

P.-La existencia del nivel intuitivo le ayuda a explicar la conveniencia de que hablemos de nuestros enunciados morales en términos de verdad y falsedad (OE, 7.6). Por otro lado, el nivel crítico, que es puramente prescriptivo, se encarga de cuestionar la validez de los criterios que se tienen por verdaderos en el nivel intuitivo y determina si pueden ser defendidos o no. La necesidad de que esto sea así, sin embargo, parece ser puramente psicológica: necesitamos poder pensar que los enunciados morales que suscribimos son verdaderos. Pero algunos filósofos no ven ninguna necesidad aquí. Richard Rorty, por ejemplo, niega que necesitemos pensar en nuestros enunciados morales como siendo verdaderos o falsos.

$R$-Como dije antes, no he leúlo los álitmos trabajos de Rorty. Baso mi recomendación de no ascender al nivel crítico con demasiada prontitud en el reconocimiento de la fragilidad humana. Los arcángeles, a diferencia de los humanos, podrian ascender a tal nivel de un modo seguro en cualquier momento. En cierto sentido, se trata de una justificación psicológica, pero no como la que ustedes ofrecen. A pesar de Rorty, necesitamos explicar por qué la gente ha pensado que los juicios morales pueden ser verdaderos o falsos, y la forma más sencilla de hacerlo es señalar qué verdad hay en la idea 
de que pueden serlo. Hay cierta verdad en el nivel intuitivo.

P.-Aparte de ayudarle a explicar la necesidad de seguir hablando de los enunciados morales como pudiendo ser verdaderos o falsos, la distinción entre niveles de pensamiento moral le ayuda a solucionar también el problema del acrático y el satanista: puesto que ambos se mueven en el nivel intuitivo tan sólo, los dos pueden comprender la verdad de los juicios morales, pero actuar contrariamente a lo que éstos prescriben. Ahora bien, esta respuesta parece trasladar el problema simplemente al nivel intuitivo...

R.-El problema con el acrático y el satanista es que no piensan a nivel critico. Esto se debe a que no han hecho suficiente filosofia moral. El problema con aquellos que usan estos personajes como argumentos es que no son conscientes de la distinción entre los dos niveles del pensamiento moral.

P.-Consideremos ahora su utilitarismo. En su opinión, la aplicación de la lógica del lenguaje moral al mundo conduce finalmente a la adopción de una forma peculiar de utilitarismo con base kantiana o utilitarismo de la voluntad racional: la lógica del lenguajc moral disciplina el pensamiento moral de tal modo que un agente moral tan sólo puede aceptar o querer consistentemente aquellos cursos de acciones que pueden ser universalmente prescritos, y eso equivale a querer aquellos cursos de acciones que maximizarán la satisfacción de preferencias de todas las personas implicadas, independicntemente de los puestos que éstas ocupen. ¿Cómo situaría su utilitarismo en una taxonomía de teorias utilitaristas? Dicho de otro modo, ¿qué posición ocupa su utilitarismo con respecto a los debates clásicos dentro del utilitarismo: con respecto al debate entre el utilitarismo del acto y el utilitarismo de la regla; con respecto al debate entre el principio de utilidad como un criterio de deci- sión y el principio de utilidad como un criterio de lo correcto?

R.-Mi utilitarismo es a la vez un utílitarismo de la regla y un utilitarismo del acto. La tesis de la universalizabilidad hace que todos los juicios morales sean instancias de reglas; pero lo que se juzga es el acto, en toda su particularidad, de acuerdo con estas reglas. En mi libro Freedom and Reason (capitulo 7, «Utilitarianism») me ocupé ya de estas cuestiones, anticipando asi el tratamiento que posteriomente les dio David Lyons. Mi teoria, por otro lado, ofrece tanto criterios de decisión como criterios de to correcto. Ofrece un procedimiento para realizar decisiones correctas. Este procedimiento es, en esencia, el mismo que el de Kant.

P.-Tal sería, pues, el lugar de su teoría en relación con los debates clásicos del utilitarismo. Pero, icómo se comporta su versión ante algumas objeciones clásicas contra el mismo? Por ejemplo, ide qué modo hace frente a la objeción según la cual el utilitarismo es una teoría demasiado exigente para el individuo y que amenaza su integridad?

R.-Ustedes tienen en mente seguramente la objeción planteada por Bemard Williams. En opinión de Williams es absurdo pedir a un hombre que deje de lado sus proyectos personales a fin de atenerse a los cálculos utilitaristas. Pero entonces la humildad cristiana y la agape y sus contrapartes humanistas son peticiones absurdas también (aunque ello es poco destacable). Lo que es más destacable, sin embargo, es que defina uintegridad como la persecución centrada en uno mismo de proyectos propios y que considere un error por parte del utilitarismo el que pueda estar en conflicto con ella. /Essays in Ethical Theory, Oxford Clarendon Press, 1989, p. 219n.] Hitler es un ejemplo perfecto de la aintegridads de que habla Willams. Con respecto a la objeción de que el utilitarismo 
es demasiado exigente la solución se halla en la division entre niveles. El nivel crítico prescribira la adopción de aquellos principios intuitivos que llevarân a las personas a hacerlo lo mejor posible, teniendo en cuenta las capacidades de cada una de ellas. Tales principios serán exigentes, pero no demasiado exigentes.
P.-Aún nos queda una última pregun*. ta, obligada en este caso. ¿Cómo ve el futuro de la filosofía moral? ¿Cree que la situación ahora es mejor que cuando usted empezó?

$R$-Podria ser mejor si los filósofos prestaran atención y comprendieran lo que he escrito. 